\section{Comentarios a «Estudio de prevalencia de retinopatía diabética en pacientes diabéticos mediante retinógrafo no midriático»}

\section{Commentaries to the «Use of non mydriatic retinography to determine the prevalence of diabetic retinopathy in diabetic patients»}

Apreciado Director de Archivos de la SEO.

En relación al artículo de los autores Vleming et al (1) titulado «Estudio de prevalencia de retinopatía diabética en pacientes diabéticos mediante retinógrafo no midriático», he de felicitar primero a los autores ya que poner en marcha una unidad de cribado de la retinopatía diabética mediante cámara no midriática, es un trabajo importante y muchas veces poco agradecido, además desarrollar un estudio epidemiológico es asimismo difícil de llevar a cabo en nuestros Hospitales con el grado de presión asistencial que habitualmente tenemos, y finalmente si el estudio incluye un número elevado de pacientes, como es el presente caso (1.393 pacientes) aún tiene más mérito, y lo dice quien ha llevado a cabo diversos estudios epidemiológicos y es conocedor del tema. No obstante quisiera hacer una serie de indicaciones sobre el presente estudio, los autores encuentran una prevalencia de retinopatía diabética de un $21 \%$, cifra que dicen ser inferior a los estudios publicados en nuestro país. Efectivamente la prevalencia de retinopatía ha ido descendiendo desde los primeros estudios publicados, así Barahona et al en 1985 encontraba una prevalencia de un $53,3 \%$ (2), cifra acorde con los estudios publicados durante unas décadas, entre ellos el nuestro (3) referenciado por los autores y que encontraba una prevalencia de un $39,05 \%$ en 1996, posteriores estudios realizados en nuestra área (4) descendieron la cifra de prevalencia hasta valores situados entre $24,4 \%$, cifra similar a la de otros autores como Goldaracena et al (5) con un $23,5 \%$.

Todas estas cifras se pueden justificar por varios factores: en primer lugar ha aumentado el diagnóstico de la diabetes mellitas por parte de los Médicos de atención Familiar y Comunitaria, al ir extendiéndose por nuestra geografía el actual modelo de Atención Primaria de Salud, además no hemos de olvidar que en el año 1998 Organización Mundial de la Salud (OMS) modificó los criterios diagnósti- cos y de clasificación de la diabetes mellitus, pasando del valor $140 \mathrm{mg} / \mathrm{dL}$ de glucosa como cifra límite en ayunas a $126 \mathrm{mg} / \mathrm{dL}$, este hecho ha incluido un gran número de nuevos pacientes diabéticos y finalmente hemos también de tener en cuenta que en el mundo occidental también existe un incremento de pacientes diabéticos independiente del aumento de la edad de la población, situando la prevalencia de diabetes mellitas en una cifra de un $10 \%$ de la población, cuando antes se situaba solo en el 5\%. En conjunto pues todos estos factores hacen disminuir la prevalencia de retinopatía diabética.

De todas maneras encuentro a faltar en el estudio una referencia a la población total dependiente del Área 3 de la Comunidad de Madrid, y a su vez el valor de los diabéticos censados en las Áreas Básicas de Salud del Área correspondiente, cifra que actualmente todas la ABS disponen, y que en el presente estudio podría sernos de utilidad para comparar los resultados obtenidos. Quisiera además preguntar a los autores si el número de pacientes cribados mediante la cámara no midriática incluye los controlados habitualmente en el Hospital de referencia por el Servicio de Oftalmología, ya que en muchas ocasiones, estos pacientes ya no pasan por las unidades de cámara no midriática, esto explicaría el bajo índice pacientes con formas severas de retinopatía y la ausencia de pacientes con retinopatía proliferativa.

No quiero finalizar sin felicitar de nuevo a los autores por el presente estudio, que no olvidemos hace referencia a la utilidad de la cámara no midriática en el cribado de los pacientes diabéticos, y que en el futuro inmediato mejorará la calidad de vida de los mismos sin ninguna duda.

Quisiera también invitar a los mismos y a otros posible interesados en un estudio a nivel Nacional para una mejor determinación epidemiológica, de un patología tan importante como es la retinopatía diabética, ya que un estudio multicéntrico utilizando las unidades de cámara no midriática actualmente en funcionamiento pueden ayudarnos mucho a conocer la salud ocular de nuestros pacientes diabéticos.

Dr. Pedro Romero-Aroca Jefe de Servicio de Oftalmología Hospital Universitario Sant Joan de Reus

(Tarragona)

romeropere@gmail.com promero@grupsagessa.com 


\section{BIBLIOGRAFÍA}

1. Vleming EN, Castro M, López-Molina MI, Teus MA. Estudio de prevalencia de retinopatía diabética en pacientes diabéticos mediante retinógrafo no midriático. Arch Soc Esp Oftalmol 2009; 84: 231-236.

2. Barahona JM, Alio JL. Epidemiologia y prevención de la ceguera por diabetes mellitus en nuestro medio. Arch Soc Esp Oftalmol 1985; 48: 27-46.

3. Romero P, Del Castillo D. Estudio de prevalencia de retinopatía diabe tica en la población del Baix Camp (Tarragona). Arch Soc Esp Oftalmol 1996; 71: 261-268.

4. Romero Aroca P, Calviño Domínguez O, del Castillo Déjardin D. Estudio epidemiológico de retinopatía diabética, en una área básica de salud. Arch Soc Esp Oftalmol 2000; 75: 147-152.

5. Goldaracena MB, Escudero JM, Arrondo A, Vilarubia A, Aramendia $B$, Iturralde $R$. Prevalencia de retinopatía diabética en una población diabética registrada en atención primaria. Arch Soc Esp oftalmol 1998; 73: 263-268.

Réplica

Apreciado Director de Archivos de la SEO.

En respuesta a la carta al director realizada por el Dr. Pedro Romero-Aroca quería agradecer al mis- mo los acertados comentarios acerca de la prevalencia de la retinopatía diabética en nuestro medio y su evolución a lo largo del tiempo.

La población del área 3 de Madrid en 2008 era de 379.000 habitantes y estimamos el número de diabéticos en 19.000. Los pacientes controlados en el Hospital no fueron evaluados por el estudio disminuyendo por lo tanto la prevalencia de retinopatías diabéticas severas y proliferativas de nuestro estudio. Sin embargo, dado el carácter prospectivo de nuestro estudio, parece lógico incluir sólo a los pacientes a partir de una fecha determinada. Nuestra idea es considerar esos pacientes, ya diagnosticados y en seguimiento, cuando el número de pacientes de nuestra base de datos prospectiva se acerque al $100 \%$ de la población diabética de nuestra área de salud.

Atentamente,

\section{Eduardo N}

Licenciado en Medicina. Servicio de oftalmología. Hospital Universitario Príncipe de Asturias. Alcalá de Henares. Madrid. España. E-mail: e_vleming@yahoo.es 